Research Article

\title{
Circulating Dengue Serotypes in Central Delhi during 2015 Outbreak
}

\author{
Kumar S Abhishek, Naresh Kumar ${ }^{2}$, Anita Chakravarti ${ }^{3}, \underline{C P}$ Baveja $^{4}$ \\ ${ }^{1}$ Senior Resident, Department of Microbiology, AlIMS, Jodhpur, Rajasthan, India. \\ ${ }^{2}$ Professor, Department of Medicine, Maulana Azad Medical College, New Delhi, India. \\ ${ }^{3}$ Professor and Head, Department of Microbiology, SGT Medical College Hospital and Research Institute, Gurugram, Haryana, \\ India. \\ ${ }^{4}$ Director Professor, Department of Microbiology, Maulana Azad Medical College, New Delhi, India. \\ DOI: https://doi.org/10.24321/2349.7181.202001
}

\section{I $\quad \mathbf{N} \quad \mathbf{F} \quad \mathbf{O}$}

\author{
Corresponding Author: \\ Kumar S Abhishek, Department of Microbiology, \\ AlIMS, Jodhpur, Rajasthan, India. \\ E-mail Id: \\ get.abhi1904@gmail.com \\ Orcid Id: \\ https://orcid.org/0000-0001-7019-4478 \\ How to cite this article: \\ Abhishek KS, Kumar N, Chakravarti A, Baveja CP. \\ Circulating Dengue Serotypes in Central Delhi \\ during 2015 Outbreak. J Adv Res Med 2020; \\ 7(1): 1-4. \\ Date of Submission: 2020-04-16 \\ Date of Acceptance: 2020-07-07
}

\section{$\begin{array}{llllllllllllll}\mathbf{A} & \mathbf{B} & \mathbf{S} & \mathbf{T} & \mathbf{R} & \mathbf{A} & \mathbf{C} & \mathbf{T}\end{array}$}

Introduction: Dengue fever is caused by an arthropod borne dengue virus. Delhi has witnessed various outbreaks in the last two decades. All the four dengue serotypes are being reported to circulate during each outbreak with predominance of one or more. Each year the prevalent circulating serotype changes. This study was planned to identify the predominant circulating dengue serotype during 2015 Delhi outbreak.

Material and Methods: Venous blood was collected from the 150 suspected adult patients attending the outdoor department of medicine. Dengue fever was diagnosed using NS-1 antigen ELISA and dengue serotypes were detected using conventional RT-PCR among 15 randomly selected NS-1 antigen positive sera.

Result: Out of 150 suspected cases, 44 were positive for dengue NS-1 antigen. RT-PCR among 15 positive sera detected five DEN-1, three DEN-2 and seven DEN-3 serotypes.

Conclusion: The prevalent circulating dengue serotype in central Delhi during 2015 outbreak was DEN-3 followed by DEN-1.

Keywords: Dengue Serotypes, Delhi, Outbreak, NS-1 Antigen, RT-PCR

\section{Introduction}

Dengue fever is caused by an arthropod borne Dengue virus. It's a member of flavivirus belonging to family Flaviviridae and transmitted primarily by the bite of female Aedes aegypti mosquito in Delhi. ${ }^{1}$ It presents with wide spectrum of signs and symptoms ranging from acute febrile illness to fatal complicated dengue in form of dengue hemorrhagic fever and dengue shock syndrome. It's a major health concern among tropical and sub-tropical countries including India. As per estimate, $\mathbf{3 9 0}$ million dengue virus infection occurs across the globe annually, of which around 96 million manifests clinically. ${ }^{2}$ As per WHO, number of dengue cases have increased over 15 folds over last two decades. ${ }^{3}$

In India, it was first reported in 1946 and from then it is endemic in various states including Delhi. ${ }^{4}$ Delhi has witnessed various outbreaks during past two decades like 2003, 2006, 2010, 2013 and 2015..$^{5}$ Aedes agypti the prime vector in India, breeds in stagnated clean water in contrast to other mosquito species. ${ }^{6}$ Dengue virus has four closely related serotypes namely DEN-1, DEN-2, DEN-3 and DEN-4, though the fifth variant has been isolated, but its occurrence in India is not well documented. ${ }^{7}$ During an 
outbreak, all the four serotypes are found to circulate with predominance of one or more.

The pathogenesis of severe dengue is not well understood, though antibody dependent enhancement hypothesis is widely accepted in cases of secondary dengue. ${ }^{8}$ Dengue infection triggers both Th- 1 and Th- 2 types of immune response, resulting in secretion of various pro- and antiinflammatory cytokines. These secreted cytokines play an important role in the pathogenesis of dengue. Detection of raised Interleukins (mainly IL-4 and IL-10) could be an important tool for early detect possible severe dengue cases. ${ }^{9}$ Since, infecting serotypes plays an important part in the development of severe dengue, it becomes important to know the circulating serotypes. Thus, this study was planned during later half of 2015 to identify the predominant circulating dengue serotype(s) in central Delhi and to prime the treating physician about the risk of development of severe dengue among secondary dengue cases arising due to change in circulating dengue serotypes both intra-city and inter-epidemics.

\section{Material and Methods}

This study was carried out in collaboration with the department of Medicine and Microbiology at Maulana Azad Medical college, New Delhi.

Five milliliters of venous blood was collected in plain vial under proper aseptic conditions from the 150 dengue suspected adult patients visiting outdoor patient department of Medicine having history of fever for $1-5$ days (acute phase illness). Serum was separated, aliquoted and immediately transferred to $-70^{\circ} \mathrm{C}$ until processed further.

\section{Dengue Confirmation}

Dengue infection was confirmed by NS-1 antigen detection using NS-1 Ag Microlisa kits (J. Mitra and Co. Pvt. Ltd., New Delhi) as per manufacturer's instructions.

\section{Detection of Viral Serotypes}

Randomly fifteen NS-1 antigen positive acute phase sera were selected for serotyping using conventional Reverse Transcriptase Polymerase Chain Reaction (RT-PCR).

Viral RNA was extracted using high pure viral RNA extraction kit (Invitrogen, PureLink ${ }^{\circledR}$, USA) as per manufacturer's instructions. The extracted RNA template was reverse transcribed into complimentary DNA (cDNA) using gene specific primers D1 and D2. Table 1 and 2. Dengue virus serotypes were detected by subjecting RT-PCR product of 511bp to nested PCR using D1 and TS1, TS2, TS3 and TS4 primers. ${ }^{10}$ Table 3 and 4 .

The amplicon thus obtained were separated electrophoretically on $2 \%$ agarose gel containing ethidium bromide and visualized under ultra violet (UV) light using Gel Doc system Figure 1.

M: Molecular ladder 50bp, 1 - 5: Type specific amplicon from samples.

This study was approved from the Institution Ethical Committee and written informed consent was obtained from the patients.

Table I.RT-PCR Primer sequences ${ }^{10}$

\begin{tabular}{|c|c|}
\hline Primers & Size in base pair (bp) \\
\hline D15'TCAATATGCTGAAACGCGCGAGAAACCG-3' & 511 \\
\hline D25'TTGCACCAACAGTCAATGTCTTCAGGTTC-3' & 511 \\
\hline
\end{tabular}

Table 2.RT-PCR cycle

\begin{tabular}{|c|c|}
\hline Preincubation & $94 \circ \mathrm{C}$ for $3 \mathrm{~min}$ \\
\hline Denaturation $\times 35$ cycles & $94{ }^{\circ} \mathrm{C}$ for $30 \mathrm{sec}$ \\
\hline Primer annealing $\times 35$ cycles & $55^{\circ} \mathrm{C}$ for $1 \mathrm{~min}$ \\
\hline Primer extension $\times 35$ cycles & $72^{\circ} \mathrm{C}$ for $2 \mathrm{~min}$ \\
\hline Final extension & $72^{\circ} \mathrm{C}$ for $10 \mathrm{~min}$ \\
\hline
\end{tabular}

Table 3.Serotype specific nested PCR primers ${ }^{10}$

\begin{tabular}{|c|c|}
\hline Primers & Size in base pair (bp) \\
\hline TS1 5'-CGTCTCAGTGATCCGGGGG-3' & 482 \\
\hline TS2 5'-CGCCACAAGGGCCATGAACAG-3' & 119 \\
\hline TS3 5'-TAACATCATCATGAGACAGAGC3' & 290 \\
\hline TS4 5'-CTCTGTTGTCTTAAACAAGAGA-3' & 392 \\
\hline
\end{tabular}


Table 4.Serotype specific nested PCR cycle

\begin{tabular}{|c|c|}
\hline Preincubation & $\mathbf{9 4}=\mathrm{C}$ for $\mathbf{3} \mathrm{min}$ \\
\hline Denaturation $\times 35$ cycles & $94 \circ \mathrm{C}$ for $30 \mathrm{sec}$ \\
\hline Primer annealing $\times 35$ cycles & $55 \circ \mathrm{C}$ for $1 \mathrm{~min}$ \\
\hline Primer extension $\times 35$ cycles & $72 \circ \mathrm{C}$ for $2 \mathrm{~min}$ \\
\hline Final extension & $72 \circ \mathrm{C}$ for $10 \mathrm{~min}$ \\
\hline
\end{tabular}

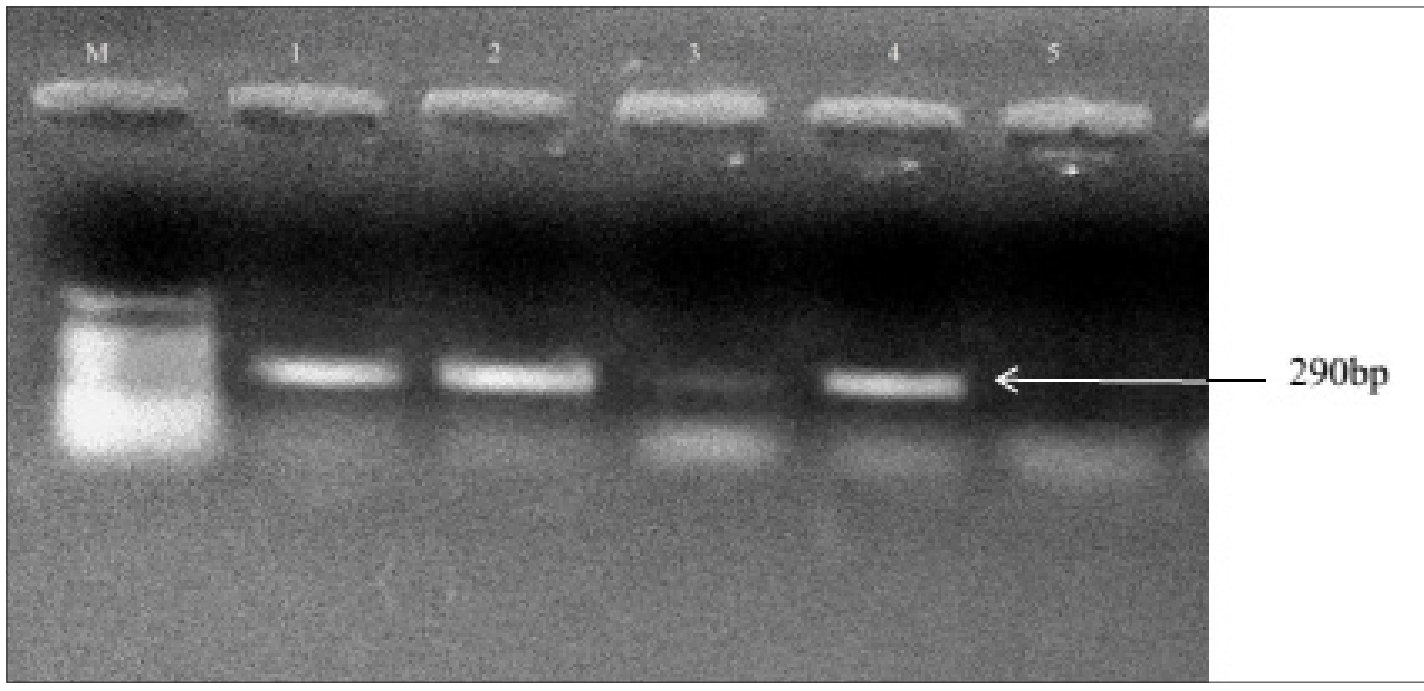

Figure I.The agarose gel image showing dengue serotype specific nested PCR amplicon. (DEN-3 290 bp band)

\section{Result}

A total of 150 suspected cases of acute dengue (fever history of 1-5 days) were included in this study during July - December 2015. Out of these 150 suspected cases, forty-four (29.3\%) were positive for NS-1 dengue antigen. RT-PCR was performed on 15 randomly selected NS-1 positive sera. Among these infection with DEN-3 was found in seven, DEN-1 in five and DEN-2 in three patients, while DEN-4 serotype was not detected in any of the sera.

\section{Discussion}

Delhi witnessed one of the major dengue outbreak during 2015 and contributed 15,867 cases with 60 deaths. ${ }^{11}$ Every year during monsoon and early winter, Delhi faces a huge number of dengue cases which is attributed to expanding population and urbanization. ${ }^{12}$ It has been reported that more than $25 \%$ of the population in Delhi have a past history of dengue infection and thus, percentage of secondary dengue cases is also increasing. ${ }^{13,14}$ The frequency of dengue outbreaks is on rising trend from the past two decades. Although it's difficult to comment why the incidence are increasing, but various associated factors can be attributed like rainfall, stagnated water in pots, coolers, discarded tyres, construction sites. aquarium, and many more, lack of preventive measure and immune status of the pollution to prevalent dengue serotype.

Circulating dengue serotype changes almost every year. During 2012 co-dominance of DEN-2 and DEN-3 was seen in Delhi, while during 2013 and 2015, dominance of DEN-2 was seen. ${ }^{15,16}$ Similarly, all the four serotypes were found to be circulating with predominance of DEN-2 along with concurrent infection with multiple serotypes during 2015. ${ }^{17}$ In contrast, our study from central Delhi reveals predominance of DEN-3 followed by DEN-1. Thus, clearly demonstrating that the predominant serotypes might vary even within city. Since, secondary infection with different serotype may lead to severe complicated dengue mostly with DEN-2 and DEN-3 followed by DEN-1 and DEN-4 serotypes, it becomes important to know the prevalent dengue serotype circulating during each outbreak and also among different areas within the city. ${ }^{18}$

Moreover, dengue and chikungunya co-infection is not so uncommon. ${ }^{19}$ Mortality associated with dengue and chikungunya co-infection has also been reported. ${ }^{20}$ Since Aedes mosquito is a common vector for both dengue and chikungunya, there is always a high possibility of transmitting the infection simultaneously. Once the mosquito is infected with dengue virus, it remains infective for life. Furthermore, the whole progeny of mosquito gets infected as they demonstrate vertical transmission (trans- 
ovarian) of dengue virus. ${ }^{21}$ Thus, breeding site control is the main component of vector control measures.

\section{Conclusion}

During 2015 Delhi outbreak, co-circulation of more than one serotypes was observed. In central Delhi, predominant circulating dengue serotype was DEN-3 followed by DEN1 unlike other studies. Thus, there is always a possibility of predominance of different serotype in different areas of same city during the same outbreak. So, it becomes important to identify the predominant circulating serotype both from different part of the city and inter-epidemics. Thus, priming the treating physicians about the risk of development of severe dengue among secondary dengue cases previously infected with different dengue serotype and timely initiation of treatment in the line of severe/ complicated dengue. As scientists across the globe is still looking for an effective antiviral agent against dengue virus, breeding site control remains an important component of vector control measures.

\section{Conflicts of Interest: None}

\section{References}

1. Kumar V, Nagpal BN, Pande V, Srivastava A, Gupta SK, Paul R et al. Comparison of Ae. aegypti breeding in localities of different socioeconomic groups of Delhi, India. Int J Mosq Res 2015; 2(2): 83-88.

2. Bhatt $S$, Gething PW, Brady OJ, Messina JP, Farlow AW, Moyes CL et al., The global distribution and burden of dengue. Nature 2013; 496(7446): 504-507.

3. WHO. Dengue and severe dengue (fact sheet). Available at: http://www.who.int/mediacentre/factsheets/fs117/ en/. [Accessed 11 April 2020, n.d.].

4. Karamchandani PV. Dengue group of fevers in India. Lancet 1946; 247(6386): 92-93.

5. Gupta E, Mohan S, Bajpai M, Choudhary A, Singh G. Circulation of Dengue virus1 (DENV1) serotype in Delhi, during 201011 after Dengue virus3 (DENV3) predominance: A single centre hospitalbased study. $J$ Vector Borne Dis 2012; 49: 8285.

6. Sunyoto T, Bhatia R, Dash AP. Changing epidemiology of dengue in South-East Asia. WHO South East Asia J Public Health 2013; 2(1): 23-27.

7. Mustafa MS, Rasotgi V, Jain S, Gupta V. Discovery of fifth serotype of dengue virus (DENV5): A new public health dilemma in dengue control. Med J Armed Forces India 2015; 71: 6770.

8. Halstead SB. Pathogenesis of dengue: Challenges to molecular biology. Science 1988; 239: 476481.

9. Abhishek KS, Chakravarti A, Baveja CP, Kumar N, Siddiqui O, Kumar S. Association of interleukin-2,- 4 and-10 with dengue severity. Indian J Pathol Microbio 2017; 60(1): 66-69.
10. Lanciotti RS, Calisher CH, Gubler DJ, Chang GJ, Vorndam AV. Rapid detection and typing of dengue viruses from clinical samples by using reverse transcriptase polymerase chain reaction. J Clin Microbiol 1992; 30(3): 545-551.

11. Available from: http://www.nvbdcp.gov.in/dencd.html. [Accessed 11 April 2020].

12. Chakravarti A, Suresh K, Neha, Shweta, Malik S. Dengue outbreak in Delhi in 2009: study of laboratory and clinical parameters. J Commun Dis 2012; 44(3): 163-168.

13. Vikram K, Nagpal BN, Pande V et al. An epidemiological study of dengue in Delhi, India Acta Trop 2016; 153: 21-27.

14. Siddiqui O, Chakravarti A, Abhishek KS. Dengue: Lessions of an Outbreak. J Clin Diagn Res 2016 Jun; 10(6): DC01-4.

15. Sharma P, Mittal V, Chhabra M, Singh P, Bhattacharya $D$, Venkatesh S. Dominance shift of DENV-1 towards reemergence and co-dominant circulation of DENV-2 \& DENV-3 during postmonsoon period of 2012 in Delhi, India. J Virol Retrovirol 2014; 1(1): 1-5.

16. Islam A, Abdullah M, Tazeen A, Afreen N, Deeba F, Naqvi IH et al. Detection of all the four serotypes of dengue virus in New Delhi, India during post monsoon season of 2015. Indian J Health Sci Care 2016; 3(3): 24-29.

17. Savargaonkar D, Sinha S, Srivastava B et al. An epidemiological study of dengue and its coinfections in Delhi. Int J Infect Dis 2018; 74: 41-46.

18. Fried JR, Gibbons RV, Kalayanarooj S et al. Serotypespecific differences in the risk of dengue hemorrhagic fever: an analysis of data collected in Bangkok, Thailand from 1994 to 2006. PLoS Negl Trop Dis 2010; 4(3):e617.

19. Abhishek KS, Chakravarti A. Simultaneous detection of IgM antibodies against dengue and chikungunya: Coinfection or cross-reactivity? J Family Med Prim Care 2019; 8: 2420-2423.

20. Mercado M, Acosta-Reyes J, Parra E et al. Clinical and histopathological features of fatal cases with dengue and chikungunya virus co-infection in Colombia, 2014 to 2015. Euro Surveill 2016; 21(22): 1-6.

21. Chen LH, Wilson ME. Non-vector transmission of dengue and other mosquito borne flaviviruses. Dengue Bulletin 2005; 29: 13-31. 